\title{
Prototype Design and Development of Flex Sensor Controlled Bionic Arm for the Handicapped Person
}

\author{
Md. Mehedi Rahman \\ Rana \\ Computer Science and \\ Engineering Discipline \\ Khulna University \\ Khulna, Bangladesh
}

\author{
Md. Farhan Zaman \\ Department of Computer \\ Science and Engineering \\ Northern University of \\ Business and Technology \\ Khulna \\ Khulna, Bangladesh
}

\author{
Shuvo Kumar Ray \\ Department of Computer \\ Science and Engineering \\ Northern University of \\ Business and Technology \\ Khulna \\ Khulna, Bangladesh
}

\author{
Jabed Al Faysal \\ Computer Science and \\ Engineering Discipline \\ Khulna University \\ Khulna, Bangladesh
}

\begin{abstract}
Increasing industrialization coupled with lack of awareness of safety parameters is contributing to rising amputation levels. The search for safer, simpler, and automated prosthetic arms is growing day by day. Continuous efforts are being made to design and develop prosthetic arms. But the price range has always been an issue. On the other hand, with the increasing amount of Industrialization, the necessity of robotic hand is also increasing. Also, if we look at the military technology, then using combat drones for reconnaissance and attacking over the opponent has given an extra advantage in the field of military warfare. With keeping in mind, the following scenarios this project has been developed as the primary prototype of a prosthetic arm. This hand can perform various tasks including moving and lifting the objects. However, servo motors are used to control the robotic hand. These motors' movement can be performed at any angle when needed. These servo motors are attached with Arduino while the robotic hand has been connected with jumper wire through breadboard with Arduino board. To manage the hand's function, we used two approaches: one is a flex sensor and another one is muscle signals extracted from finger movement. The robotic hand can be used for a variety of activities, including bomb dispersal, humanitarian assistance, and surgery.
\end{abstract}

\section{Keywords}

Robotic Arm, Flex Sensor, Servo Motor, Arduino UNO R3.

\section{INTRODUCTION}

In this rapidly evolving technological age, the term robotics is getting quite frequent and the technology behind robotics is developing day by day. The reason behind using robotics in a frequent format is to increase life's effectiveness, reduce human labor, and save time. Due to amputation people are losing their limbs every day. The alarming fact is due to the expensiveness of robotic arms, it is out of reach for many lower-income people. This gesture control robotic arm is built in such a way that it would be affordable to the majority of people. This arm is part of a complete future robot. The design will allow the robotic hand to perform various functions including bomb disposal, radioactive material handling, sensitive task handling, etc. The robotic hand can perform simple tasks such as positioning and picking up items that are positioned far away from the user [1].

In general, there are two different ways to deal with robotic arms; wired and wireless. The benefit of the wired system is that it doesn't need batteries, which is needed in wireless technology. But the downside is that it is difficult to maintain because there are far-ranging cables. External interference occurs in wireless systems, while wired ones have less of it. Again, wired systems are far less expensive than wireless systems [1]. Many applications depend on the technology for robotic hands as medical science, industrial uses, military uses, and educational purposes. We have developed a design and developed a robotic hand connected and performs its movement with servo motors. It has been designed as a fourfinger grip. As for the humanoid robot, the generation of human-like motions has already been deployed and has proven popular [2]. A robotic arm having four degrees of freedom is programmed to pick up and position items of a given weight. Servomotors (with a torque of $11 \mathrm{~kg}$ ) are used to assist in lifting the objects. [3]. The combination of flex sensor and servo has completed the process for developing a robotic arm.

\section{RELATED WORKS}

There have been several types of research in the area of humanoid robots over the last decade, resulting in the discovery of several systems for controlling these robots. In [4], it is proposed to develop a mechanical hand that mimics human hand using flex sensors and servo engines. Another study demonstrates an instructive concept, as automatic control is an energizing and challenging area of science [5]. The significant achievement of this work is that every human arm moment can be precisely projected onto the robotic arm. Furthermore, programming simplicity of the microcontroller has reduced the challenge [6]. The research on the use of resistive flex sensors, considering their working standards, fabrication considerations, electrical characteristics, and working chemical principles, was able to cover the front-end aspects, material features, and physics-based models [7]. The development of the robotic arm is continuing with the help of MATLAB and Simulink. Servo motors are used as actuators on the robotic arm. Cables that imitate the tendons of the human arm are used to guide the finger motions [8].

\section{SYSTEM ARCHITECTURE}

An effective and simple design has been introduced to develop a robotic arm. For this reason, Arduino is used as a micro-controller. Here, Arduino will be used to process the data and controlling the hand. The robotic hand includes all joints that are comparable to the hand of a human, which play an inevitable part in coordinated finger movement, giving the ability to grab different shapes. Also used a flex sensor as a pressure sensor and which will be used to collect the data from the bending of the finger. The breadboard has been used 
for the extension of connection. Jumper wires are used for establishing the connection. Servo motors have used for controlling the grip opening and closing of the palm. The system architecture is represented in Fig. 1.

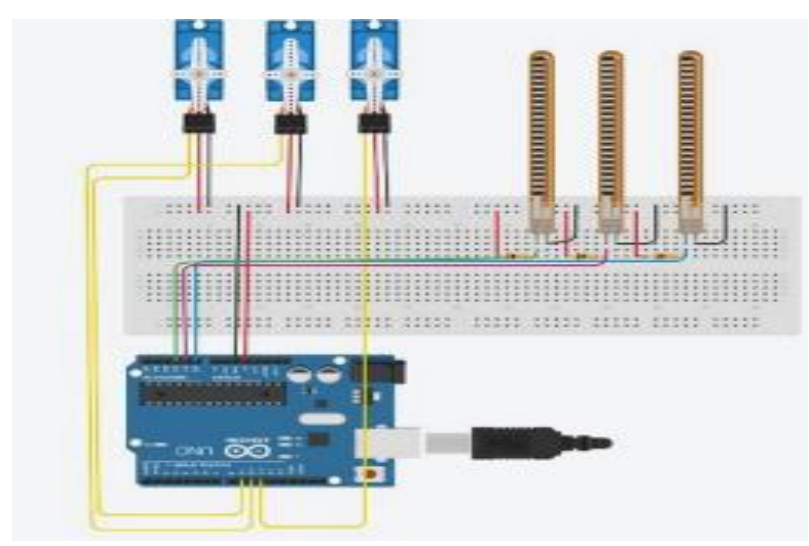

Fig. 1: The complete architecture of the system

\subsection{Mechanical Design}

The mechanical design part plays a significant role here in this project. A board have been used to develop the hand for creating a low-cost prototype. Here, Arduino will be used for and as a micro-controller for controlling the robotic hand. All the joints of the robotic hand are comparatively similar to a human hand that gives the hand the advantage of free movement and gripping capability. To build the prototype for cost-effectiveness, three flex sensors are used to collect data from bending by creating resistivity and three servos have been used for controlling five fingers. The servos help the hand to open and close the fingers. The prototype of the hand is in Fig. 2.

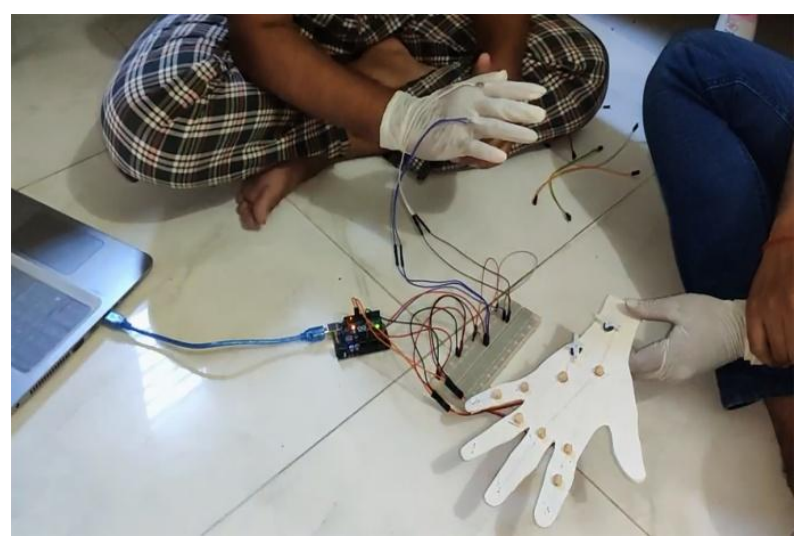

Fig. 2: Prototype of robotic hand

\subsection{Main Control Unit}

The Main Control Unit (MCU) of the project is Arduino. Arduino Uno is used for performing the following tasks:

(1) Flex sensor movement detection: Three flex sensors are being used to detect the bend of fingers. This project utilizes a basic flex sensor with a length of 2.2". The Flex Sensor is entirely constructed of resistive carbon components referred to as substrates or velostats. As the velostat is bent, it generates a resistance related to the curve range; the resistive value increases as the radius decreases. The flex sensor is in Fig. 3.

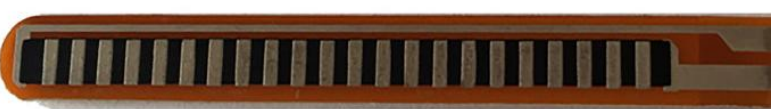

Fig. 3: Flex sensor

All these flex sensor connections are made directly to the voltage source's ground terminal. A 10k resistor is used to connect the positive terminal of the voltage source to the second flex sensor link. The second flex sensor link is also attached to the Arduino's analog channels. Arduino receives and processes analog data from the flex sensor. Table 1 illustrates the connections between the flex sensor and Arduino.

Table 1. Connection details between micro-controller and flex sensors

\begin{tabular}{|c|c|}
\hline Fingers with Flex Sensor & Arduino Connections \\
\hline Index & A0 \\
\hline Middle & A1 \\
\hline Ring & \\
\hline Little & A2 \\
\hline Thumb & An \\
\hline
\end{tabular}

(2) Controlling robotic hand: Three servo motors are used to regulate and control the finger's movement. The MCU or Arduino is used to power these servo motors. Arduino analyzes the data obtained at the receiver and then instructs the servo motors to act appropriately. Earlier in Fig. 1, the relation between servo motors and Arduino was shown. Using the breadboard, the VCC connections of all the servo motors are wired directly to the positive terminal. Similarly, all of the servos' GND links have been directly wired to the negative terminal through a breadboard. The servos' signal pins have been connected to the Arduino UNO R3's digital pin ports. These digital pin ports can control the servos' shaft rotation. The Servos and MCU signal pin references are shown in Table 2.

Table 2. Signal pin connections of servos with me ports

\begin{tabular}{|c|c|}
\hline Signal Pin Connections & Arduino Connections \\
\hline Index & D10 \\
\hline Middle & D11 \\
\hline Ring & D9 \\
\hline Little & Thumb \\
\hline
\end{tabular}

\section{OPERATION AND RESULT} ANALYSIS

The procedures used to guide the robotic hand are described below. We used a Flex sensor to monitor the robotic hand's speed. Three flex sensors are used to track each finger's movement. These sensors are integrated into the glove's palm. The stretching of the sensors detects the rotation of the 
fingertips. Then, using the analyzed data, we can monitor the movement of the robotic hand's fingers through the servos. These flex sensors are wired to a ten-thousand-ohm resistor and are connected to an Arduino. Each flex sensor is connected to a $10 \mathrm{~K}$ resistor in this configuration. The voltage around each flex sensor is computed in both the straight and bending positions. Table 3 shows the voltage shift in the flex sensor of each finger where Fig. 4 indicates the graphical view of voltage changing.

Table 3. Voltage Change for Flex Sensor

\begin{tabular}{|c|c|c|}
\hline $\begin{array}{c}\text { Flex Sensor attached } \\
\text { with fingers }\end{array}$ & $\begin{array}{c}\text { Voltage with } \\
\text { straight flex } \\
\text { sensor }(\mathbf{V})\end{array}$ & $\begin{array}{c}\text { Voltage with } \\
\text { full bend flex } \\
\text { sensor }(\mathbf{V})\end{array}$ \\
\hline Thumb & 1.80 & 2.36 \\
\hline Index & 2.15 & 3.30 \\
\hline Ring & 1.875 & 2.5 \\
\hline
\end{tabular}

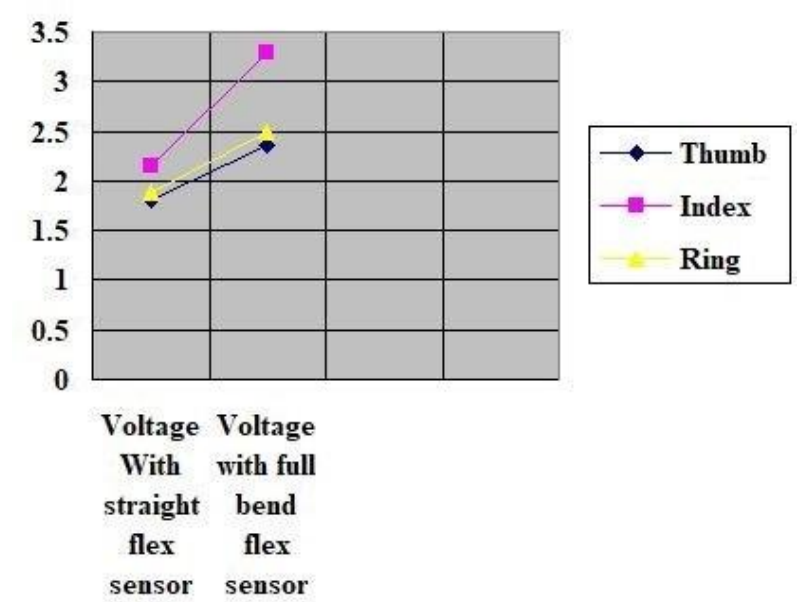

Fig. 4: Graphical view of voltage changing

Then, using the serial display, we observed the analog values of straight and bend flex sensors. When the flex sensor was straight, the servo direction was shown in degree form. The results are shown in Fig. 5.

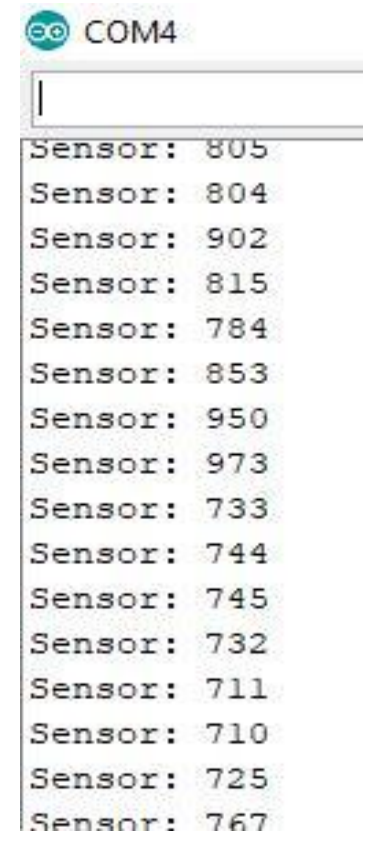

Fig. 5: Numerical findings on a serial display for a straight flex sensor and bended flex sensor

Two initial tasks are conducted to evaluate the robotic hand: opening and closing the fingers. The experiment is conducted by opening and closing both of the human hand's fingers; the robotic hand mirrored the human hand's action when wearing a glove. Fig. 6 and Fig. 7 show the opened and closed robotic hands.

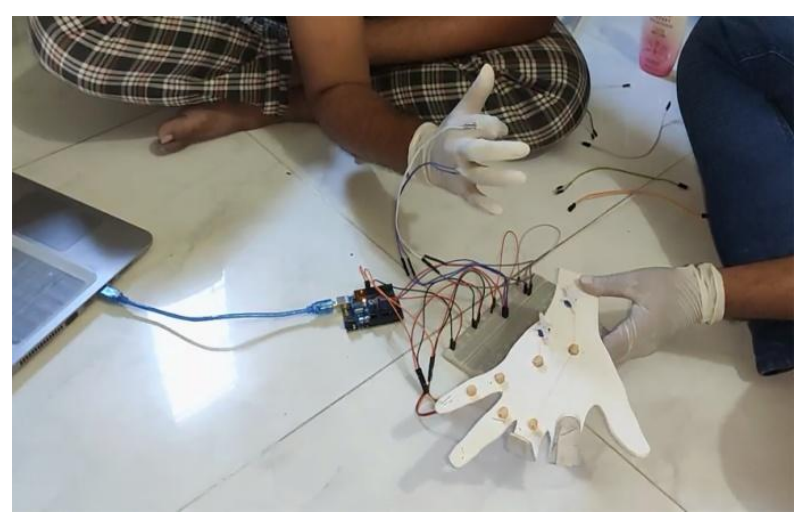

Fig. 6: Robotic hand is open as all fingers are open

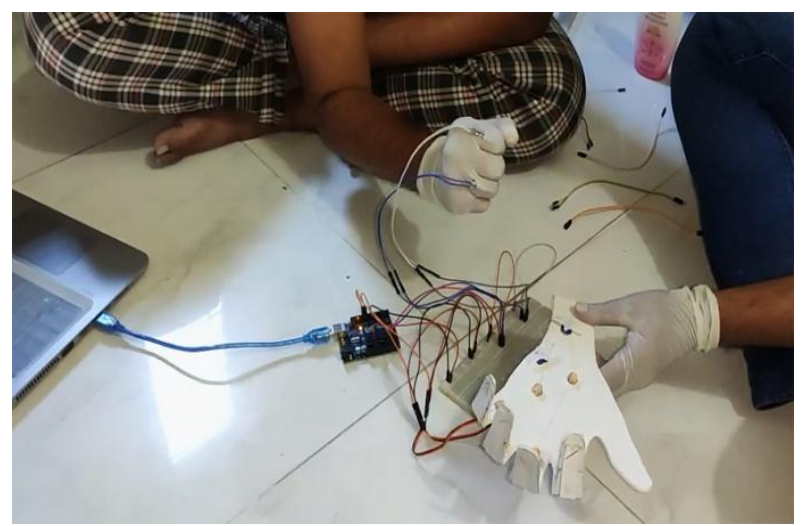

Fig. 7: Robotic hand is closed as all fingers are closed 
There are some vulnerabilities related to IoT devices that are connected to Wireless, these devices are related to healthcare products. Some of the products poses the threat of CVE-20205902, CVE-2020-3635, CVE-2020-16257 and CVE-20203671 [9]. But the most dangerous problem is CVE-202025654 pacemaker. Here, the attacker can use this vulnerability to bypass the security of the pacemaker. By manipulating the command of pacemaker, the attacker can change the configuration of it. Most of the IoT based devices are affected with some kind of vulnerabilities. As the myo arm band follows the similar technology which is a wireless technology that can be hacked and accessed from outside. This is an alarming issue. Myo arm band or myoware also possess some other issues including adaptation difficulty, Bluetooth technology requirement, and limited functionality. Additionally, unwanted behavior might occur at times. It has also proven that Bluetooth and other wireless technologies can be affected by electromagnetic fields. So, if myoware is used, there is a possibility that it can be affected by a magnetic field or emp blast.

\section{CONCLUSIONS}

This paper proposed a sample prototype of a robot for people who have lost their upper limbs. The robot's motions are driven by the guidance given by humans. The arrangement of Arduino with cable connection made the prototype costefficient. Arduino-UNO is a microcontroller that has high processing power. By using Arduino UNO's powerful computing capability, the objective of grasping and holding the objects has been accomplished. The robot possesses an extraordinary capacity to operate in a highly dynamic environment. It is capable of lifting objects weighing up to one kilogram $(\mathrm{kg})$. Handicapped people are capable of performing their everyday household works independently. As a result, the permanent need for human assistance for disabled persons has been eliminated to a certain extent by the use of this robot. A flex sensor-based robotic arm is more accurate than many of its competitors. It is also humanfriendly and cost-effective as well. In the future, we will work on some modifications and add some additional features which can significantly improve its efficiency and make it capable of using in industries and healthcare sectors.

\section{REFERENCES}

[1] Latif, Shahid, et al. "Design and development of muscle and flex sensor controlled robotic hand for disabled persons." 2019 International Conference on Applied and Engineering Mathematics (ICAEM). IEEE, 2019.

[2] Rahman, Md Anisur, et al. "Design, analysis and implementation of a robotic arm-the animator." American Journal of Engineering Research (AJER) 2.10 (2013): 298-307.

[3] Krishna, R., Bala, G. S., ASC, S. S., Sarma, B. B. P., \& Alla, G. S. (2012). "Design and implementation of a robotic arm based on haptic technology." Int. J. of Eng. Research and Applications, 2(34).

[4] J. Y. Hande, N. Malusare, and H. D. S. Sawarbandhe, "Design for Robotic Hand Using Flex-sensor." International Journal of Advanced Research in Electronics and Communication Engineering (IJARECE): 2846-2850.

[5] Shen, Zhong, et al. "A soft stretchable bending sensor and data glove applications." Robotics and biomimetics 3.1 (2016): 1-8

[6] Meshram, Urmila Devendra, and R. Harkare. "Fpga based five axis robot arm controller." IEEE Conference. 2005.

[7] Saggio, Giovanni, et al. "Resistive flex sensors: a survey." Smart Materials and Structures 25.1 (2015): 013001 .

[8] Afzal, Waseem, et al. "Gesture control robotic arm using flex sensor" Applied and Computational Mathematics 6.4 (2017): 171-176.

[9] Sidhpurwala, Huzaifa. "Oss-sec: CVE-2020-25654 Pacemaker: ACL Restrictions Bypass." Sec. Accessed March 11, 2021. https://seclists.org/oss-sec/2020/q4/83. 